\title{
THE PROPER ORTHOGONAL DECOMPOSITION IN THE ANALYSIS OF TURBULENT FLOWS
}

Gal Berkooz, Philip Holmes, and John L. Lumley

Cornell University, Ithaca, New York 14853

KEY WORDS: coherent structures, empirical eigenfunctions, modeling, turbulence

\section{INTRODUCTION}

\subsection{The Problems of Turbulence}

It has often been remarked that turbulence is a subject of great scientific and technological importance, and yet one of the least understood (e.g. McComb 1990). To an outsider this may seem strange, since the basic physical laws of fluid mechanics are well established, an excellent mathematical model is available in the Navier-Stokes equations, and the results of well over a century of increasingly sophisticated experiments are at our disposal. One major difficulty, of course, is that the governing equations are nonlinear and little is known about their solutions at high Reynolds number, even in simple geometries. Even mathematical questions as basic as existence and uniqueness are unsettled in three spatial dimensions (cf Temam 1988). A second problem, more important from the physical viewpoint, is that experiments and the available mathematical evidence all indicate that turbulence involves the interaction of many degrees of freedom over broad ranges of spatial and temporal scales.

One of the problems of turbulence is to derive this complex picture from the simple laws of mass and momentum balance enshrined in the NavierStokes equations. It was to this that Ruelle \& Takens (1971) contributed with their suggestion that turbulence might be a manifestation in physical 
space of a strange attractor in phase space. Since 1971 we have witnessed great advances in dynamical-systems theory and many applications of it to fluid mechanics, with, alas, mixed results in turbulence--despite the attractive notion of using deterministic chaos in resolving the apparent paradox of a deterministic model (Navier-Stokes) that exhibits apparently random solutions. This is due not solely to the technical difficulties involved: Proof of global existence and a finite-dimensional strange attractor for the 3-D equations in a general setting would be a great mathematical achievement, but would probably be of little help to specific problems in, say, turbomachinery. For a start, rigorous estimates of attractor dimension (Téman 1988) indicate that any dynamical system which captures all of the relevant spatial scales will be of enormous dimension. Advances in such arcas will most probably nccessitate a dramatic reduction in complexity by the removal of inessential degrees of freedom.

The first real evidence that this reduction in complexity might be possible for fully developed turbulent flows came with the experimental discovery of coherent structures around the outbreak of the second world war, documented by J. T. C. Liu (1988). The existence of these structures was probably first articulated by Liepmann (1952), and was thoroughly exploited by Townsend (1956). Extensive experimental investigation did not take place until after 1970, however (see Lumley 1989). Coherent structures are organized spatial features which repeatedly appear (often in flows dominated by local shear) and undergo a characteristic temporal life cycle. The proper orthogonal decomposition, which forms the subject of this review, offers a rational method for the extraction of such features. Before we begin our discussion of it, a few more general observations on turbulence studies are appropriate.

\subsection{Experiments, Simulations, Analysis, and Understanding}

In analytical studies of turbulence, two grand currents are clear: statistical and deterministic. The former originates in the work of Reynolds (1894). The latter is harder to pin down; linear stability theory is felt to have little to do with turbulence. Nonlinear stability, however, and such things as amplitude equations, definitely are relevant, so perhaps L. D. Landau and J. T. Stuart should be credited with the beginnings of an analytical nonstatistical approach. Lorenz' work was certainly seminal. Over the past twenty years a third stream has emerged and grown to a torrent which threatens to carry everything in its path: computational fluid dynamics.

Both analytical approaches have drawbacks. Statistical methods, involving averaged quantities, immediately encounter closure problems (Monin \& Yaglom 1987), the resolution of which, even in sophisticated renormalization group theories (cf McComb 1990) usually requires use of 
empirical data (Tennekes \& Lumley 1972). Nonetheless, they are intended for and are used for fully developed turbulence. Analytical methods have so far been unable to deal with the interaction of more than a few unstable modes, usually in a weakly nonlinear context, and thus have been restricted to studies of transition or pre-turbulence. Most of the dynamical systems studies have been limited to this area. Computational fluid dynamics bypasses the shortcomings of these methods by offering direct simulation of the Navier-Stokes equations. However, unlike analysis, in which logical deductions lead stepwise to an answer, simulation provides little understanding of the solutions it produces. It is more akin to an experimental method, and no less valuable (or less confusing) for the immense quantity of data it produces, especially at high spatial resolution.

Proper orthogonal decomposition (POD), while lacking the broad sweep of the approaches mentioned above, nonetheless has something to offer all three of these. 1. It is statistically based - extracting data from experiments and simulations. 2 . Its analytical foundations supply a clear understanding of its capabilities and limitations. 3. It permits the extraction, from a turbulent field, of spatial and temporal structures judged essential according to predetermined criteria and it provides a rigorous mathematical framework for their description. As such, it offers not only a tool for the analysis and synthesis of data from experiment or simulation, but also for the construction, from the Navier-Stokes equations, of lowdimensional dynamical models for the interaction of these essential structures. Thus, coming full circle, we have a statistical technique that contributes to deterministic dynamical analysis.

In Sections 3 and 4 we review applications of the proper orthogonal decomposition, after developing its key features in Section 2. The latter is necessarily mathematical in style and while space limitations preclude a complete treatment, we include some of the new and lesser known results. Proofs are omitted; see Berkooz (1991b, Chapter 2) for details. Section 5 explores relations to some other techniques used in turbulence studies and Section 6 contains a concluding discussion. The remainder of this introductory section contains an historical survey.

\subsection{The Proper Orthogonal Decomposition}

The proper orthogonal decomposition is a procedure for extracting a basis for a modal decomposition from an ensemble of signals. Its power lies in the mathematical properties that suggest that it is the preferred basis to use in many circumstances. The POD was introduced in the context of turbulence by Lumley (1967, ef 1981). In other disciplines the same procedure goes by the names Karhunen-Loève decomposition or principal components analysis and it seems to have been independently rediscovered 
several times, cf Sirovich (1987). According to Lumley, quoting A. M. Yaglom (personal communication), the POD was suggested independently by several scientists, e.g. Kosambi (1943), Loève (1945), Karhunen (1946), Pougachev (1953), and Obukhov (1954). For use of the POD in other disciplines see: Papoulis (1965)_random variables; Rosenfeld \& Kak (1982) — image processing; Algazi \& Sakrison (1969) — signal analysis; Andrews et al (1967)-data compression; Preisendorfer (1988)-oceanography; and Gay \& Ray $(1986,1988)$ - process identification and control in chemical engineering. Introductory discussions of the method in the context of fluid mechanical problems can also be found in Sirovich (1987, 1989, 1990) and Holmes (1990).

The attractiveness of the POD lies in the fact that it is a linear procedure. The mathematical theory behind it is the spectral theory of compact, selfadjoint operators. This robustness makes it a safe haven in the intimidating world of nonlinearity; although this may not do the physical violence of linearization methods, the linear nature of the POD is the source of its limitations, as will emerge from what follows. However, it should be made clear that the POD makes no assumptions about the linearity of the problem to which it is applied. In this respect it is as blind as Fourier analysis, and as general.

\section{FUNDAMENTALS OF THE PROPER ORTHOGONAL DECOMPOSITION}

\subsection{The Eigenvalue Problem}

For simplicity we introduce the proper orthogonal decomposition in the context of scalar fields: (complex-valued) functions defined on a interval $\Omega$ of the real line. The interval might be the width of the flow, or the computational domain. We restrict ourselves to the space of functions which are square integrable (or, in physical terms, fields with finite kinetic energy) on this interval. We need an inner product $(f, g)=\int_{\Omega} f(x) g^{*}(x) d x$, and a norm $\|f\|=(f, f)^{1 / 2}$. We start with an ensemble of realizations of the function $u(x)$, and ask which single (deterministic) function is most similar to the members of $u(x)$ on average? We need an averaging operation \langle\rangle , which may be a time, space, ensemble, or phase average. We suppose that the probabilistic structure of the ensemble is such that the average and limiting operations can be interchanged (cf Lumley 1971). Mathematically, the notion of "most similar" corresponds to seeking a function $\phi$ such that

$$
\max _{\psi}\left\langle|(u, \psi)|^{2}\right\rangle /(\psi, \psi)=\left\langle|(u, \phi)|^{2}\right\rangle /(\phi, \phi)
$$


That is, we find the member of the $\psi(=\phi)$ which maximizes the (normalized) inner product with the field $u$, which is most nearly parallel in function space. This is a classical problem in the calculus of variations. A necessary condition for (2.1) to hold is that $\phi$ is an eigenfunction of the two-point correlation tensor

$$
\int_{\Omega}\left\langle u(x) u^{*}\left(x^{\prime}\right)\right\rangle \phi\left(x^{\prime}\right) d x^{\prime}=\lambda \phi(x) .
$$

We define the average $R\left(x, x^{\prime}\right)=\left\langle u(x) u^{*}\left(x^{\prime}\right)\right\rangle$. That the maximum in (2.1) is achieved, and corresponds to the largest eigenvalue $\lambda_{1}$ of (2.2) is a consequence of spectral theory (Reisz \& Sz. Nagy 1955). Moreover HilbertSchmidt theory assures us that there is not one, but a denumerable infinity of solutions of (2.2), as long as $\Omega$ is bounded. We will call these the empirical eigenfunctions, and denote them by $\left\{\phi_{k}\right\}$ and normalize them so that $\left\|\phi_{k}\right\|=1$. Note that the subscript $k$ does not denote a vector, but a member of the sequence. We order the eigenvalues by $\lambda_{i} \geq \lambda_{i+1}$, observing that the non-negative definiteness of $R\left(x, x^{\prime}\right)$ assures that $\lambda_{i} \geq 0$. We also have a diagonal decomposition:

$$
R\left(x, x^{\prime}\right)=\Sigma_{k} \lambda_{k} \phi_{k}(x) \phi_{k}^{*}\left(x^{\prime}\right) .
$$

In (2.3) and hereafter the sum is from 1 to infinity unless explicity indicated otherwise. As we will see in Section 2.2, almost every member (in a measure sense) of the ensemble may be reproduced by a modal decomposition in the eigenfunctions:

$$
u(x)=\Sigma_{k} a_{k} \phi_{k}(x) \text {. }
$$

The diagonal representation of the two-point correlation tensor $R$ ensures that the modal amplitudes are uncorrelated:

$$
\left\langle a_{k} a_{k}^{*}\right\rangle=\delta_{k k} \lambda_{k} ;
$$

see Section 2.3 below. Here (2.4) is the proper orthogonal decomposition, and the set $\left\{\phi_{k}\right\}$ is called an empirical basis.

\subsection{The Span of the Empirical Basis}

The first step in understanding what can be done with the sequence $\left\{\phi_{k}\right\}$ is to characterize the set $S=\left\{\left.\Sigma a_{i} \phi_{i}|\Sigma| a_{i}\right|^{2}<\infty\right\}$, which is the span of the set $\left\{\phi_{i}\right\}$. That is, what functions can be represented by convergent sequences of empirical eigenfunctions? Note that we retain only eigenfunctions with nonzero eigenvalues, so that the $\left\{\phi_{k}\right\}$ need not form a complete basis. If one adds all the eigenfunctions with zero eigenvalue, one obtains a 
complete basis, but loses some of the advantages of the POD, as we shall see.

The first proposition describes the ability of the empirical basis to reconstruct the ensemble from which it was generated. The propositions are stated a little loosely, and we do not provide proofs here; tighter statements and proofs may be found in Berkooz (1991b).

Proposition 2.1 If $R\left(x, x^{\prime}\right)$ is continuous, then almost every member $u$ of the ensemble belongs to $S$.

We denote by $X$ the subset of $u$ 's of the ensemble that belongs to $S$, i.e. where Proposition 2.1 holds.

Corollary 2.1 Let $\left\{b_{i}\right\}$ be an infinite sequence of real numbers, and $u_{i}$ an infinite sequence of ensemble members in $X$. If $v=\Sigma_{i} b_{i} u_{i}$ is square integrable on $\Omega$, then $v$ lies in $S$.

The following proposition together with the corollary above will characterize $S$.

Proposition 2.2 If $\theta$ is in $S$, then there exist infinite sequences $\left\{u_{i}\right\}$ in $X$ and scalars $\left\{b_{i}\right\}$ such that $\theta(x)=\Sigma_{i} b_{i} u_{i}$.

We thus have a complete characterization of the span of the eigenfunctions: It is exactly the span of all the realizations of $u(x)$, with the exception of a set of measure zero. In particular, with the exception of a set of measure zero, every member of the ensemble that generated the eigenfunctions can be represented in terms of the eigenfunctions. A special case of this result (when the $u_{i}$ take on discrete values, as would be the case in a computer experiment) was observed independently in Aubry et al (1991a).

From this we see that the sequence $\left\{\phi_{i}\right\}$ need not be complete. It is complete only if one includes the kernel of the operator $R$, that is, all the (generalized) eigenfunctions with zero eigenvalues. Of course, if $R$ is positive definite, there are no zero eigenvalues, and one does get a complete basis. However, in many applications one can argue on physical grounds that the realizations $u(x, t)$ do not span the space of square integrable functions on $\Omega$; for example, see Section 2.4 below. This result highlights a strong property of the POD. It a priori limits the space studied to the smallest linear subspace that is sufficient to describe the observed phenomena. This can be stated as a corollary:

Corollary 2.2 If all the square integrable functions $u$ on $\Omega$ having a certain property form a closed linear subspace, then the empirical eigenfunctions have the same property, and the converse is also true. 
The classical example is incompressibility. If $R\left(x, x^{\prime}\right)$ is formed from realizations of divergence-free vector fields $u$, then the eigenfunctions $\left\{\phi_{i}\right\}$ are also divergence-free.

\subsection{Optimality}

Suppose we have a signal $u(x, t)$ and a decomposition with respect to an (arbitrary) orthonormal basis $\left\{\psi_{i}\right\}$ :

$$
u(x, t)=\Sigma_{i} b_{i}(t) \psi_{i}(x)
$$

If the $\left\{\psi_{i}\right\}$ have been nondimensionalized and normalized to give $\left(\psi_{i}, \psi_{j}\right)=\delta_{i j}$, then the coefficients $b_{i}$ carry the dimension of the quantity $u$. If $u(x, t)$ is a velocity, the average kinetic energy per unit mass over the experiment is given by

$$
\int_{\Omega}\left\langle u u^{*}\right\rangle d x=\Sigma_{i}\left\langle b_{i} b_{i}^{*}\right\rangle .
$$

Hence, we may say that $\left\langle b_{i} b_{i}^{*}\right\rangle$ represents the average kinetic energy in the $i$-th mode. The following proposition establishes what is called the optimality of the POD or Karhunen-Loève decomposition.

Proposition 2.3 Let $u(x, t)$ be an ensemble member square integrable on $\Omega$ for almost every $t$ and $\left\{\phi_{i}, \lambda_{i}\right\}$ be the POD orthonormal basis set with associated eigenvalues. Let

$$
u(x, t)=\Sigma_{i} a_{i}(t) \phi_{i}(x)
$$

be the decomposition with respect to this basis, where equality is almost everywhere. Let $\left\{\psi_{i}\right\}$ be an arbitrary orthonormal set such that

$$
u(x, t)=\Sigma_{i} b_{i}(t) \psi_{i}(x) .
$$

Then the following hold:

1. $\left\langle a_{i}(t) a_{j}^{*}(t)\right\rangle=\delta_{i j} \lambda_{i}$, i.e. the POD coefficients are uncorrelated.

2. For every $n$ we have $\Sigma_{i}^{n}\left\langle a_{i}(t) a_{i}^{*}(t)\right\rangle=\Sigma_{i}^{n} \lambda_{i} \geq \Sigma_{i}^{n}\left\langle b_{i}(t) b_{i}^{*}(t)\right\rangle$.

This proposition is the basis for the claim that the POD or KarhunenLoève decomposition is optimal for modeling or reconstructing a signal $u(x, t)$. It implies that, among all linear decompositions, this is the most efficient, in the sense that, for a given number of modes the projection on the subspace used for modeling will contain the most kinetic energy possible in an average sense. In addition, the time series of the coefficients $a_{i}(t)$ are uncorrelated. 


\subsection{Symmetries and Homogeneity}

We start by describing a particular kind of symmetry. We say that the two-point correlation $R\left(x, x^{\prime}\right)$ is homogeneous or translation invariant if $R\left(x, x^{\prime}\right)=r\left(x-x^{\prime}\right)$, i.e. $R$ depends only on the difference of the two coordinates. In general, homogeneity of a system is defined through multipoint moments, but we only need second-order moments here. Assuming $\Omega$ is bounded and $u$ is periodic, we may develop $r$ in a Fourier series,

$$
r\left(x-x^{\prime}\right)=\Sigma C_{k} e^{2 \pi i k\left(x-x^{\prime}\right)} .
$$

One can then solve the eigenvalue problem via the unique representation

$$
R\left(x, x^{\prime}\right)=\Sigma C_{n} e^{2 \pi i n x} e^{-2 \pi i n x^{\prime}},
$$

which implies that the $e^{2 \pi i n x}$ are exactly the eigenfunctions with eigenvalues $C_{n}$. Conversely, if the eigenfunctions are Fourier modes we can write (2.11) which implies (2.10). In summary, we can state

Proposition 2.4 $R\left(x, x^{\prime}\right)=r\left(x-x^{\prime}\right)$ if and only if the eigenfunctions of $R$ are Fourier modes.

This observation is especially useful in systems where the domain $\Omega$ is of higher dimension. For example, if $\Omega$ is $2-\mathrm{D}$, then we have

$$
R\left(\mathbf{x}, \mathbf{x}^{\prime}\right)=R\left(x, y, x^{\prime}, y^{\prime}\right),
$$

and if the $x$-direction is homogeneous, the problem of finding eigenfunctions in a 2-D domain is decoupled into a set of 1-D problems by writing

$$
R=R\left(x-x^{\prime}, y, y^{\prime}\right)
$$

and performing the same procedure as above, yielding a 1-D eigenvalue problem for every Fourier wavenumber. Examples of such applications can be found in Herzog (1986), Moin \& Moser (1989); also see Lumley (1971). The observations above can be generalized to other cases where part of the domain has a more general symmetry group structure, see Berkooz \& Titi (1992).

Unfortunately, in the context of coherent structures in the turbulent boundary layer, the above observation leads to Fourier structures which are, of course, not localized in all space directions, unlike the events observed. In an attempt to avoid this, and reintroduce locality, it is necessary to introduce phase relationships among the Fourier modes. In the following treatment, we adapt Lumley's application of the shot-noise decomposition (Rice 1944; Lumley 1971, 1981). Imagine a building block, which is the basic coherent structure, and a process that sprinkles the units 
randomly on the real line. (See Figure 1.) If $f(x)$ is the building block, with 0 as a reference point, in order to move the structure so that its reference point is at $y$ we perform the convolution

$$
u(x)=\int \delta(\xi-y) f(x-\xi) d \xi,
$$

where $\delta(\xi)$ is the Dirac delta function (working in the space of generalized functions or distributions). This prompts us to the following:

Definition 2.1 A convolution of the type

$$
u(x, t)=\int g_{\mathrm{t}}(\xi) f(x-\xi) d \xi,
$$

where $g_{\mathrm{t}}(\xi)$ is a random process in the space of generalized functions, will be called a shot-noise decomposition of $u(x, t)$.

The goal is to reconstruct $f$ from statistics of the system. To develop intuition for Definition 2.1, assume for simplicity that both $f$ and $u$ have an upper bound on frequencies in their Fourier decompositions, and that they are periodic. Then $g$ need not be a generalized function, and the Fourier transform of $g$ will be well-defined. If $u, g$, and $f$ are the Fourier transforms of $u, g$, and $f$ respectively, then clearly $\mathrm{u}=\mathrm{gf}$. We see that, in general, a shot-noise decomposition is always possible, and that moreover
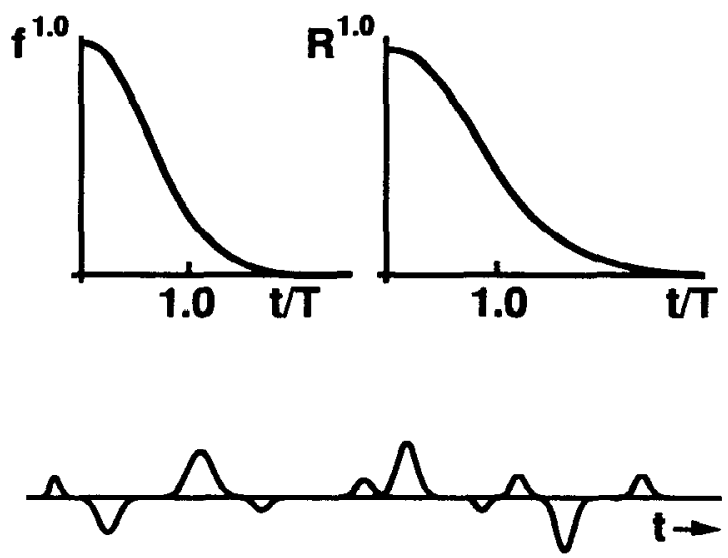

Figure 1 (Top) The basic building block and its autocorrelation. (Bottom) The result of random sprinkling with Gaussian amplitudes. 
it is far from unique; one also has freedom in choosing $f$ and $g$. To remove the ambiguity in the decomposition, and to formalize the notion that $g$ "randomly" sprinkles $f$ 's, we make the following assumption on the process $g$.

Assumption 2.1 Leg $g$ be uncorrelated in nonoverlapping intervals, i.e. $\left\langle g(\xi) g\left(\xi^{\prime}\right)\right\rangle=\delta\left(\xi-\xi^{\prime}\right)$.

This assumption removes part of the ambiguity of the decomposition, as follows:

Proposition 2.5 If $\mathrm{R}$ is the Fourier transform of the two-point velocity correlation for a homogeneous process, then $\mathrm{R}=|\mathrm{f}|^{2}$.

We see that Assumption 2.1 prescribes the power spectrum of the building block; the phase angles are yet to be determined. Before we discuss the retrieval of the phase information, we will examine some physical aspects of the shot-noise decomposition with the above assumption. It seems that this approach formalizes rather well the stochastic sprinkling of structures in physical space. An extension of this formalism to include stochastic sprinkling in time is also possible, the assumption being extended to noncorrelatedness in time as well as in physical space. The structure of the building block in time corresponds to the life cycle, or evolution, of the coherent structure. The assumptions of noncorrelatedness in physical space and in time are, of course, simplistic approximations; coherent structures cannot be too close to each other, in either space or time, and hence there must be a short-range correlation of $g$. In addition, adjacent coherent structures affect each other dynamically, and hence $g$ must be statistically dependent at different places and times, even if uncorrelated. In addition, the assumption of a single building block may also be restrictive, since we may expect to meet more than one form of coherent structure. In multiple dimensions, we should include the possibility that the building blocks will occur with different orientations. Some of these deficiencies will come back to haunt us when we try to retrieve the phase information for $f$.

A rational procedure was suggested by Lumley $(1971,1981)$ to obtain the phase information from the bi-spectrum (Brillinger \& Rosenblatt 1967, Lii et al 1976). Our goal is to find the phase angle of the Fourier coefficients of $f$. We already have the moduli. We want to find $\theta(\kappa)$ such that

$$
\mathbf{f}=\mathrm{R}^{1 / 2} e^{2 \pi i \theta(\kappa)} .
$$




$$
\begin{aligned}
& \left\langle u(x) u\left(x+r_{1}\right) u\left(x+r_{2}\right)\right\rangle=\iiint f(x-\xi) f\left(x+r_{1}-\xi^{\prime}\right) \\
& \times f\left(x+r_{2}-\xi^{\prime \prime}\right)\left\langle g(\xi) g\left(\xi^{\prime}\right) g\left(\xi^{\prime \prime}\right)\right\rangle d \xi d \xi^{\prime} d \xi^{\prime \prime}
\end{aligned}
$$

We now extend the assumption on $g$ and require that it be triply uncorrelated on nonoverlapping intervals, thus obtaining for the right hand side

$$
\int f(x) f\left(x+r_{1}\right) f\left(x+r_{2}\right) d x .
$$

If we designate the triple correlation in $(2.17)$ by $B\left(r_{1}, r_{2}\right)$ and its Fourier transform by $\mathrm{B}\left(\kappa_{1}, \kappa_{2}\right)$, we obtain

$$
\begin{gathered}
\mathrm{B}\left(\kappa_{1}, \kappa_{2}\right)=\mathrm{R}^{1 / 2}\left(\kappa_{1}\right) \mathrm{R}^{1 / 2}\left(\kappa_{2}\right) \mathrm{R}^{1 / 2}\left(\kappa_{1}+\kappa_{2}\right) \\
\quad \times \exp \left\{2 \pi i\left[\theta\left(\kappa_{1}\right)+\theta\left(\kappa_{2}\right)-\theta\left(\kappa_{1}+\kappa_{2}\right)\right]\right\} .
\end{gathered}
$$

The known quantities are $\mathrm{B}\left(\kappa_{1}, \kappa_{2}\right)$, and $\mathrm{R}(\kappa)$. As Lumley (1971) observed (see also Moin \& Moser 1989), this problem is, in general, not solvable exactly, since $B\left(\kappa_{1}, \kappa_{2}\right)$ may not be factorable as the right hand side prescribes. In fact, if our assumption (that $g$ is triply uncorrelated) is correct, $B$ will be so factorable, and not otherwise. There is very little information on $B$ in turbulence, and none on its factorability. Moin \& Moser observe that this problem is encountered in other disciplines as well; see Bartlet et al (1984) and Matsuoka \& Ulrych (1984). Lumley suggested the following simple solution: reduce $B$ to a one-dimensional domain by looking at $\mathrm{B}(c, c \kappa)$, where $c$ is an arbitrary number, and solve the above equation on a finite number of points. This is not very convincing, however, and it is exactly at this point that our assumptions on $g$ come back to haunt us. The lack of an exact general solution to the bi-spectrum equation suggests that our assumptions may have been too simplistic, either regarding the existence of a single building block, or regarding the statistical behavior of $g$.

An alternative strategy for representing localized structures in homogeneous directions involves the use of wavelet decompositions (Meyer 1987). While not optimal (see Proposition 2.4), it has recently been shown that periodic spline wavelets (Perrier \& Basdevant 1989, cf Farge 1992) are not much less efficient than Fourier modes in capturing kinetic energy on the average. More specifically, Berkooz et al (1991a) show that, if the autocorrelation $R\left(x-x^{\prime}\right)$ is reconstructed with average error $\varepsilon$ using $N$ Fourier modes, then a wavelet reconstruction with a similar number of modes will incur error $3 c+c / 2^{\delta n}$, where $n$ is the order of the splines used, and the constants $\delta$ and $c$ depend on how the wavelet octaves relate to $N$. 
There are some restrictions on this result, and it relies on the rapid decay in Fourier space of spline wavelets, but it suggests a promising new direction to explore.

We can use the POD to examine the ergodicity of a dynamical system. Physically, if the phase space of the dynamical system is partitioned into disjoint closed regions, so that a system trajectory starting in one of these never enters the others, the system is not ergodic. Now, if a system has certain symmetries, these symmetries should appear as symmetries of the invariant measure. However, starting from a given initial condition, it is possible that the solution will not explore all the states associated with the symmetry group. If this occurs, we can say that the system is not ergodic. For example, suppose a 2-D map is invariant under the symmetry $(x, y) \rightarrow$ $(y, x)$, and has two disjoint attractors lying to either side of the $x=y$ symmetry axis (and hence individually not invariant under the symmetry). A typical realization - an orbit of this map - will explore just one attractor, and will not have the symmetry of the full system. The invariant measure concentrated on the two attractors is not ergodic because of the disjointedness, and we can see this because of the lack of symmetry. As a result, if the empirical eigenfunctions obtained from a single run of the experiment (in time) have less symmetry than the problem as a whole, we conclude that the system is not ergodic. Note that it is also possible for there to be two disjoint attractors, each of which displays the full symmetry, and hence there may be no telltale lack of symmetry to point to the lack of ergodicity. More formally, we have:

Proposition 2.6 Let $S$ be a stationary ensemble of realizations, and $m$ be the invariant measure associated with it. Let $G$ be a linear symmetry group for $S$. Then a necessary condition for $m$ to be ergodic is that, for almost every realization, each of the finite-dimensional eigenspaces corresponding to a given eigenvalue (which results from the time averages of that experiment) is invariant under $G$.

The way one would go about checking this condition in an experiment would be:

1. Perform the experiment and measure $R\left(x, x^{\prime}\right)$.

2. Obtain the $\left\{\phi_{i}\right\}$ from $R\left(x, x^{\prime}\right)$.

3. Check that every $\phi_{i}$ satisfies the symmetry condition.

(Here, for simplicity, we consider a system with distinct eigenvalues.)

Recently Aubry et al (1991b) performed numerical integrations of the Kuramoto-Sivashinsky equation and computed the POD basis. By using the results above and the calculations cited, they conclude that for certain values of the bifurcation parameter, the system is not ergodic. On the other 
hand, if one wants to assume that the system is ergodic, one may use the symmetries of the system to increase the size of the ensemble. This approach has been advocated by Sirovich (1987) and applied in many studies (e.g. Sirovich \& Park 1990). However, one should be cautious, as there are examples (Berkooz 1990b) where the partition into ergodic components is finer than the partition into symmetric components; in this case the image of the basis obtained by one experiment under a symmetry group will not produce the basis obtained by the ensemble average measure. See Berkooz \& Titi (1992) for further discussion of this point. Caution is particularly warranted in cases of small systems or special geometries. For example, in a square Rayleigh-Bénard cell there is a possibility that a preferred rotation direction of the single roll may be chosen at random at the ime of onset and may never change throughout the life of the system. This indicates that there are at least two distinct and disjoint parts for the support of the invariant measure, each associated with a rotation direction, much as in the simple map example. Similar phenomena evidently can occur in the minimal flow unit of Jimenez \& Moin (1991). The additional symmetry imposed on the ensemble of flows by artificial addition of images of flows under symmetry group elements, as advocated by Sirovich (1987), may therefore obscure the true nature of a particular system.

\subsection{The Nature of Attractors}

We first describe a geometrical consequence of the phase space description of asymptotic behavior afforded by the POD. In particular, we can give a probabilistic-geometric interpretation of the location of dynamics in phase space using Chebyshev's inequality. This generalizes the result in Foias et al (1990), who essentially reproduced the proof of Chebyshev's inequality in a specific case. Aubry et al (1991b) independently observed that the type of picture described in Foias et al (1990) is due to Chebyshev's inequality.

We will sketch the proof here, because it is geometrically instructive. First, recall Chebyshev's inequality.

Theorem 2.1 Let $\mathbf{X}$ be a vector-valued random variable with zero mean and variance $\sigma^{2}=\left\langle|\mathbf{X}|^{2}\right\rangle$. Then for any $\varepsilon>0$

$$
P\{|\mathbf{X}| \geq \varepsilon\} \leq \sigma^{2} / \varepsilon^{2}
$$

where $P\{\bullet\}$ is the probability of that event.

Now, denote by $\mathbf{X}_{n}$ the vector-valued random variable

$$
\mathbf{X}_{n}=\left\{a_{n+1}, a_{n+2}, \ldots, a_{\infty}\right\}
$$


so that the $\mathbf{X}_{n}$ have zero mean and variance $\sigma^{2}=\lambda_{n+1}+\lambda_{n+2}+\cdots$ $=\sum_{m=n+1}^{\infty} \lambda_{m}$.

Then Chebyshev's inequality gives

$$
P\left\{\left|\mathbf{X}_{n}\right| \geq \varepsilon\right\} \leq \sum_{m=n+1}^{\infty} \lambda_{m} / \varepsilon^{2} .
$$

In the space of functions $u$, square integrable on $\Omega$, the coefficients $a_{n}$ may be regarded as coordinates. The space spanned by $\left\{\phi_{1}, \ldots, \phi_{n}\right\}$ may be thought of as a surface in the function space, with associated coordinates $\left\{a_{1}, \ldots, a_{n}\right\}$. Containing this surface, and extending $\varepsilon$ on each side of it, is a slab of thickness $2 \varepsilon$, defined by $\left|\mathbf{X}_{n}\right|<\varepsilon$. The inequality (2.22) tells us how likely it is to be outside that slab. Inequality (2.22) is useless for fixed $m$ and $\varepsilon \rightarrow 0$. The way to extract something useful is to take a sequence $\varepsilon_{n} \rightarrow 0$ such that

$$
\sum_{n+1}^{\infty} \lambda_{m} / \varepsilon_{n}^{2} \rightarrow 0
$$

In other words, the $\varepsilon_{n}$ are chosen so that their squares go to zero slower than the decay of the norms of the residual modes. This will give a series of slabs with thickness going to zero, while the probability of the solutions being in those slabs goes to one.

The problem is now shifted to computing the rate of decay of the residual energy $\sum_{m=n+1}^{\infty} \lambda_{m}$. There is analytical evidence that suggests that when the POD basis is used for turbulent flows, this residual decays at least exponentially fast asymptotically, as we argue later in this section. This enables us to take a series $\varepsilon_{n}^{2} \rightarrow 0$ with a slightly smaller exponent. The result will be a series of slabs with thickness going exponentially to zero, and the probability of being in that slab going exponentially to one. This gives rise to a picture in which the attractor is very thin, albeit high or even infinite dimensional. Thus, the essentials of the dynamics may be controlled by a finite number of modes, as the dynamical models discussed in Section 4 suggest.

We turn now to a related matter. We show that if the POD spectrum decays fast enough (which is the case for systems that interest us), practically all the support of the invariant measure is contained in a compact set; that is, roughly speaking, all the likely realizations in the ensemble can be found in a relatively small set of bounded extent.

Proposition 2.7 Consider a dynamical system whose solutions are continuous and square integrable on $\Omega$. If $\lambda_{n}=O[\exp (-c n)]$, then for any $\varepsilon>0$ there exists a compact set $B_{\varepsilon}$ such that $P\left\{B_{\varepsilon}\right\}>1-\varepsilon$.

This is quite interesting. If one performs a POD decomposition on a system about which little is known a priori, and gets a discrete spectrum that decays rapidly enough (see also the next section), Proposition 2.7 allows 
us to conclude that most of the likely realizations can be found in a compact domain. It is surprising that such fundamental information can be obtained from such a simple procedure.

We next describe a very reasonable situation in which the POD spectrum is likely to fall off exponentially and thus in which Proposition 2.7 will hold. "Regularity of solutions" is a mathematical property describing, essentially, the rate of decay of the tail of the wavenumber spectrum of instantaneous solutions of a partial differential equation (PDE). It appears that solutions of many chaotic systems have very high regularity, meaning that the instantaneous wavenumber spectrum decays rapidly (exponentially in most cases) - see Promislow (1991). We note that wavenumber spectra in fluid turbulence are generally believed to fall off exponentially (see Tennekes \& Lumley 1972). We will establish here the relation between regularity results and the POD. We start by explaining what is meant by regularity, quote regularity results for the Navier-Stokes equations, and relate regularity to the eigenvalue spectrum of the POD. These results appeared in Foias et al (1990), but our treatment differs from theirs in that we use the optimality property of the POD to establish the connection, whereas they use the uncorrelatedness of the random coefficients. A closer examination of their proof shows that the estimates obtained from regularity results hold not only for the empirical bases but also for a basis of eigenfunctions of the Stokes operator or any other basis with similar orthogonality properties.

We start with regularity in a simple setting. Let the domain of our flow be a rectangular box, in which the real velocity field $\mathbf{u}$ is periodic and incompressible. We have a representation in terms of Fourier modes:

$$
\mathbf{u}(\mathbf{x}, t)=\Sigma \mathbf{u}_{\mathbf{j}}(t) e^{i \mathbf{i} \cdot \mathbf{x}} .
$$

We assume the kinetic energy is finite:

$$
\Sigma\left|\mathbf{u}_{\mathrm{j}}\right|^{2}=|\mathbf{u}|^{2} /(2 \pi)^{3}<\infty \text {. }
$$

The Stokes operator is simply $A=-\nabla^{2}$. We can define fractional powers of $A$ via

$$
A^{\alpha} \mathbf{u}=\Sigma \mathbf{u}_{\mathbf{j}}|\mathbf{j}|^{2 \alpha} e^{i \mathbf{j} \cdot \mathbf{x}} .
$$

Similarly, we can define

$$
\exp \left[\tau A^{s}\right] \mathbf{u}=\Sigma \mathbf{u}_{\mathbf{j}} \exp \left[\tau|\mathbf{j}|^{2 s}\right] e^{i \mathbf{j} \cdot \mathbf{x}} .
$$

If this sum converges, we say that $\mathbf{u}$ is Gevrey class regular. The relation between Gevrey regularity and the instantaneous turbulent spectrum is as follows. We can define a 3-D spectrum (see Tennekes \& Lumley 1972) as

$$
E(m)=\Sigma_{m \leq|j|<m+l}\left|\mathbf{u}_{\mathrm{j}}\right|^{2} .
$$


This is the energy in a spherical shell. For Gevrey class regular fields, we have

$$
\infty>\Sigma\left|\mathbf{u}_{\mathbf{j}}\right|^{2} \exp \left[2 \tau|\mathbf{j}|^{2 s}\right] \geq \Sigma E(m) \exp \left[2 \tau m^{2 s}\right] .
$$

Therefore, for Gevrey class regular velocity fields the instantaneous spectrum decays at lcast exponentially fast. As wc will see later, the last expression can be manipulated to obtain the asymptotic rate of decay of the random coefficients, which are nothing but $\left|\mathbf{u}_{\mathrm{j}}\right|^{2}$ in a Fourier representation.

To convince the reader that regularity results are useful, we must do the following: Define regularity for an arbitrary problem (not necessarily with periodic boundary conditions); present regularity results for the NavierStokes equations; and show how regularity results, which are instantaneous information, transform to average information such as a rate of decay for the POD eigenvalues.

The definition of regularity for an arbitrary domain is based on the Stokes operator. The only difference is that, instead of developing the velocity field in terms of Fourier modes, one develops it in terms of the eigenfunctions of the Stokes operator. These eigenfunctions form a complete basis. The asymptotics of the eigenvalues of the Stokes operator are the same for all reasonable domains (Constantin \& Foias 1989). Rigorous regularity results for the Navier-Stokes equations in two dimensions are given in Foias \& Témam (1989). For the 3-D Navier-Stokes equations, to simplify the discussion, we assume that the vorticity is bounded above uniformly throughout the flow and in time. This may be a very large bound, but it nevertheless needs to be assumed. This enables us to bypass the blowup problem for the Navier-Stokes equations (see Anderson et al 1984). In this case, Foias \& Témam (1989) showed that, after a transient period, there exists a $\sigma$ such that the solutions $u$ satisfy

$$
\left|A^{1 / 2} \exp \left(\sigma A^{1 / 2}\right) u\right|<C<\infty .
$$

Four remarks are in order. First, some uniform bound for the averages is essential; as shown in Berkooz (1990b), one can have an ensemble with all members exhibiting exponentially decaying tails, yet with an average spectrum not exponentially decaying. See also Novikov (1963) and Monin \& Yaglom (1987). Second, to get a comparison to the POD, one has to define some order on wavenumber space. The order we choose is through wavenumber shells, that is, through the 3-D spectrum. Third, as discussed in Berkooz (1990b) and Foias et al (1990), regularity results are relevant only to the far dissipative range of turbulence, or to very high order empirical eigenfunctions. (See Figure 2.) Fourth, the decay of the tail of the empirical eigen-spectrum will always be as fast, or faster, than that of the tail of the spectrum with respect to any other basis, in particular the 


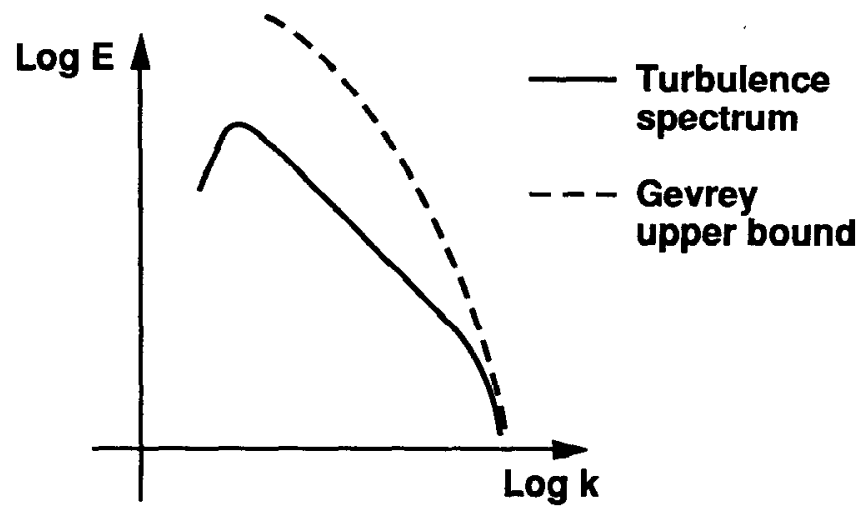

Figure 2 Spectrum of turbulence compared with upper bound for the spectrum from Gevrey regularity results.

Fourier spectrum. This is a straightforward consequence of the maximality of energy principle for the POD. (See Figure 3.)

We can now state our main results for this section.

Proposition 2.8 If a solution $\mathbf{u}(\mathbf{x}, t)$ in a domain of dimension $n$ is uniformly bounded in time in the norm $\left|A^{\alpha} \exp \left[\beta A^{\gamma}\right] \mathbf{u}\right|$ then the eigenvalues in the tail of the POD spectrum will satisfy

$$
\mu_{k}=o\left[k^{-2 \alpha / n} \exp \left(-2 \beta k^{2 \gamma / n}\right)\right] .
$$

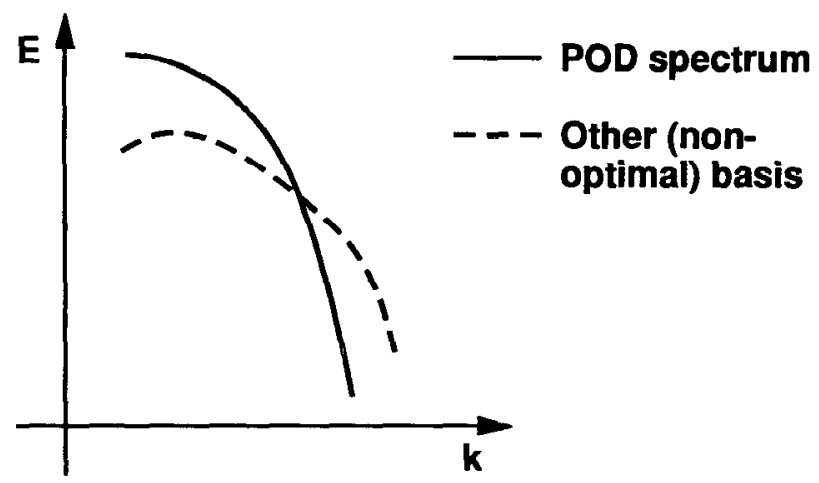

Figure 3 Typical spectrum of a dissipative system in POD and another basis. Note the "cross over" which will occur due to the equality in total energy (area under the curves). 
Proposition 2.9 Under the assumptions of the previous proposition, the empirical eigenfunctions have the same regularity as the solutions.

\subsection{Computational Schemes and Further Results}

In this section we discuss some additional points of interest regarding the POD.

THE METHOD OF SNAPSHOTS This method was proposed by Sirovich (1987). It is a numerical procedure which can save time in computation of empirical eigenfunctions. Suppose one is performing a numerical simulation on a large number of grid points $N$, the number of ensemble members deemed adequate for a description of the process is $M$, and $N \gg M$ (the fundamental question of determining $M$ is not part of Sirovich's treatment). In general the eigenfunction computation will become an $N \times N$ problem. However, this may be reduced to an $M \times M$ problem. Berkooz (1991b) gives an argument for the equivalence of the method of snapshots to the original formulation of the eigenvalue problem, as well as a linear independence condition omitted by Sirovich (1987).

DIMENSION AND THE CONDITIONAL POD An appealing concept is to define a dimension through the POD. The obvious thing to do is to define a dimension as the number of nonzero eigenvalues in the POD decomposition, as done in Aubry et al (1991a). However, this is merely the dimension of the smallest linear subspace containing the dynamics, and has, a priori, nothing to do with the Hausdorff dimension of the attractor. Even if the latter is finite, the former may not be so. This is underlined by the example in Berkooz (1990b) of a system having a limit cycle, which has Hausdorff dimension 1, with an infinite number of nonzero POD eigenvalues. Indeed, as Sirovich (1989) realized, this definition is practically useless, and he suggested the following working definition: "...the number of actual eigenfunctions required so that the captured energy is at least $90 \%$ of the total..., and that no neglected mode, on the average, contains more than $1 \%$ of the energy contained in the principle eigenfunction mode." The concept of entropy introduced by Aubry et al (1991a) based on interpreting the POD eigenvalues as probabilities is plagued by the same problem.

In Berkooz (1991b) a connection between the various dimensions is found by using the concept of a conditional POD. Suppose (for simplicity of explanation) that all the trajectories of a system are confined to a bounded region of phase space. Pick a location in that region, $u$, and consider a ball of radius $\varepsilon$ around it. Consider the POD conditioned on being in the ball. Now define a local dimension as the smallest number of conditional eigenfunctions that will return more than 1- $\delta$ of the energy. 
Now take the upper limit as $\varepsilon$ goes to zero, and then as $\delta$ goes to zero, and finally take the upper bound as $u$ ranges over the bounded region of phase space. This dimension can be shown to lie between the ordinary dimension and the Hausdorff dimension. Note that it is not obvious that this dimension should lie below the ordinary dimension; as noted in Berkooz (1990b), finite dimension of an attractor does not guarantee that there are only a finite number of energy-containing POD modes.

ASYMPTOTICS OF THE POD EIGENFUNCTIONS For the sake of completeness we mention the interesting result of Sirovich \& Knight (1985) on the asymptotic form of the POD eigenfunctions (under certain assumptions). Foias et al (1990) conclude from the results of Sirovich \& Knight (1985) that the asymptotic form of the POD eigenfunctions is that of Fourier modes. However, the results of Section 2.2 on the span of the eigenfunctions show that this cannot be completely general. Take, for example, an ensemble of realizations which are all constant on part of the domain, in which case, by Corollary 2.2 , the eigenfunctions will also have this property and so cannot be asymptotically close to Fourier modes in that region.

THE POD AND THE PDF IN FUNCTION SPACE We would like to mention the connection between the POD and the PDF in functional space. The invariant measure in functional space is an object of great interest; if one could obtain it explicitly one would have "a solution to turbulence," since all multi-point (single time) statistics would be available. From this point of view the POD is seen as the linear change of basis which turns the coordinates into uncorrelated (although probably dependent) random variables. As shown by Hopf's theory of turbulence, the characteristic functional of the PDF in functional space may be obtained by multi-point correlations (Hopf 1957). This leads us to propose a very simple model for the PDF in functional space: Using the representation

$$
u(x, t)=\sum_{k} a_{k}(t) \phi_{k}(x),
$$

we assume that the $a_{k}$ 's are independent and normally distributed with variance $\lambda_{k}, a_{k} \sim N\left(0, \lambda_{k}\right)$. While this is consistent with the picture the POD gives of the flow (the coefficients are uncorrelated and the spectrum is correct), it clearly implies a strong assumption on the modal dynamics. Nonetheless, in Section 5 we will see that this model is closely related to other statistical approaches that describe coherent motions in turbulence.

\section{POD IN DATA DESCRIPTION AND ANALYSIS}

In this and the following section we survey applications of the POD. As pointed out in the introduction, we make a distinction between applications 
of the POD to analysis of experimental results and applications of the POD to data analysis and we have grouped the studies according to the classcs of problems treated: wall-bounded flows, free shear flows, convection problems, and mathematical model equations.

\subsection{Wall-Bounded Flows}

One of the earliest applications of the POD was by Bakewell \& Lumley (1967). They measured two-point correlations of one velocity component in the wall region of a fully developed turbulent pipe flow, and reconstructed the two-point correlation tensor using incompressibility and a closure assumption. The flow is approximately homogeneous in the streamwise and cross stream directions. They computed only one eigenfunction (in addition to the mean) with no streamwise variation. They reconstructed the coherent structure using 0 as a phase relation for the homogeneous cross stream direction, and obtained a pair of counterrotating rolls. In this sense their work should be considered as a pioneering study.

Herzog performed a complete 3-D POD analysis of the wall layer of a turbulent pipe flow (Herzog 1986). Herzog had relatively low spatial resolution but very well converged statistics which enabled him to compute three significant eigenfunctions for a substantial range of wavenumber pairs in the homogeneous (cross stream and streamwise) directions. This work was a major undertaking and the first full two-point correlation data set to be measured. In his reconstruction Herzog did not apply a rational method for the reconstruction of phase angles. Herzog did measure the two-point correlation with time delay but the processed results are limited to zero time lag.

Moin \& Moser (1989), using the channel-flow data base of Kim et al (1987) obtained by direct numerical simulation of a channel flow, performed a comprehensive POD analysis. The computations employ periodic boundary conditions in the spanwise and streamwise directions and the decomposition uses Fourier modes in these directions, as was done by Herzog (see Section 2.4, above). Although it is not certain that the statistics are well converged (less than 200 realizations were used), the spatial resolution is excellent. The main thrust of their work was a systematic application of the shot-noise expansion. Moin \& Moser (1989) suggest two additional, ad hoc, methods for the determination of the phase angles. One is based on a "compactness in physical space" condition and the other based on "continuity of eigenfunctions in wavenumber." The reader is referred to Moin \& Moser (1989) for details. The results of this work are what they call "characteristic structures" which dominate the production of important statistics. 
More recently Sirovich et al (1990a) and Ball et al (1991) have also computed empirical eigenfunctions from a similar direct numerical simulation of channel flow. They took a lower Reynolds number than Moin \& Moser and, rather than assuming "compactness" and "continuity" as mentioned above to determine characteristic numbers, they extracted the temporal behavior of the coefficients $a_{k}(t)$ of the empirical eigenfunction directly. These time series show strong intermittency, as one would expect from the experimental observations of the bursting process (Kline et al 1967, Robinson 1991). In fact Sirovich et al (1990a) investigate the presence of specific structures (oblique plane waves) and their influence as triggers for the bursting events.

In the numerical channel-flow studies mentioned above, when the full channel width is taken as the domain $\Omega$ for computation of the eigenvalues, a relatively large number of eigenfunctions (counting Fourier modes) are required to capture, say, $90 \%$ of the kinetic energy on average: Ball et al give a figure of about 500 modes in reasonable agreement with Keefe et al's (1987) Liapunov dimension calculations. (Moin \& Moser also consider wall region eigenfunctions.) In this respect we stress that in Herzog's study $\Omega$ is restricted to the wall layer $(0<y+<40$ wall units), and in this region convergence is considerably faster. However, in all cases, the empirical eigenfunctions representation converges significantly faster than FourierChebyshev representations of flow in the same regions.

\subsection{Free Shear Flows}

One of the first free shear flows to be analyzed was the jet (or annular mixing layer) investigated experimentally by Glauser et al (1987) and Glauser \& George (1987a,b). In this work the jet is assumed to be approximately homogeneous in the streamwise direction (the growth of the layer was not accounted for). The main results were: $(a)$ demonstration of the effectiveness of the POD in capturing kinetic energy; (b) determination of the shape of the POD eigenfunctions (the majority of the energy being in azimuthal invariant modes); (c) the proposal of a dynamical mechanism for turbulence production based on the eigenfunctions, and emphasis of the role of nonazimuthal invariant structures in turbulence production, structures which exhibit azimuthal number selection through the POD spectrum.

Sirovich and various coworkers also investigated the jet and mixing layer using numerical simulation and experimental data (Sirovich et al 1990b; Kirby et al 1990a,b). In Sirovich et al (1990b), a conditional form of the POD was applied to the mixing-layer part of the jet, based on a correlation criterion. As a result, the approximate homogeneity in the streamwise direction was broken, resulting in a 2-D eigenfunction problem. 
The results of the POD eigenfunctions gave nice descriptions of the lobes responsible for mixing. In Kirby et al (1990b) the POD is applied to a simulated supersonic shear layer. This is again a 2-D problem. The emphasis of this study is on data compression. The time-averaging procedure eliminates interesting large time-scale dynamics. Kirby et al (1990a) is similar in spirit and results to Sirovich et al (1990b), except that Large Eddy Simulation data is used as a basis for the conditional sampling rather than experimental data.

A conditional POD was applied by Glezer et al (1989) to a forced mixing layer. They term their procedure an "extended POD."

\subsection{Convection}

The POD was applied to numerical simulation of Rayleigh-Bénard convection problems by Sirovich \& Park (1990), Park \& Sirovich (1990), and Sirovich \& Deane (1991) (cf Deane \& Sirovich 1991). In the former studies, extensive use is made of discrete symmetries of the flow domain (a rectangular box) to simplify the computations by selecting parities (even or odd) for various eigenfunction components. This is especially useful in this problem, since the domain is bounded and there are no homogeneous directions to assist in data reduction. The symmetries are also used to increase the data base over which averages are taken, as discussed in Section 2.4.

The main thrust of the more recent work is to determine scaling properties of POD eigenfunctions as a function of Rayleigh number. Some scaling properties are found. As Sirovich \& Deane (1991) point out, if such scaling does hold for asymptotically large Rayleigh numbers, it will be a considerable contribution. Using a definition of $\operatorname{dim}_{K L}$ as the number of modes required to capture $90 \%$ of the energy (cf Section 2.5) an empirical relation between the Lyapunov dimension $\operatorname{dim}_{L}$ and $\operatorname{dim}_{K L}$ is traced out.

\subsection{Mathematical Models}

The POD has been applied to the analysis of the results of several mathematical models - mostly 1-D dissipative partial differential equations. These problems are inherently simpler than real world problems and therefore provide attractive tests for the method.

Chambers et al (1988) applied the POD to simulation of Burger's equation with random forcing and showed that the resulting eigenfunctions exhibit "viscous" boundary layers near the "walls" and have an outer region essentially independent of Reynolds number. Sirovich and coworkers applied the POD to the Ginzburg-Landau equation (Rodriguez \& Sirovich 1990, Sirovich 1989) and studied the bifurcation diagram for Galerkin projections and the dependence of the eigenfunctions on an 
external control parameter such as Reynolds number. They also find that the eigenfunction dependence on parameters is relatively weak. Kirby \& Armbruster (1991) applied a conditional POD and a "moving" POD to the study of bifurcation problems in the Kuramoto-Sivashinsky equation. This approach allows the identification of traveling structures. Aubry et al (1991) have also studied the problem via a consideration of the relation of the POD to symmetry groups. Without such a procedure the statistics will be homogeneous and the eigenfunctions will be Fourier modes, as described in Section 2.4 above. The procedure applied in parts of Kirby \& Armbruster (1991) is similar to the pattern-recognition techniques described in Section 5.3. Berkooz [1991a] suggested a rigorous procedure that allows the extraction of moving structures without the need to change to a moving reference frame. This is done through the use of third-order statistics. Berkooz (1991a) also provides a procedure that determines whether the signal can be viewed as a small perturbation of a moving structure.

A 2-D model involving the incompressible Navier-Stokes equations with spatially periodic forcing (the Kolmogorov flow) was also studied by Sirovich's group (Platt et al 1991). While they did not use the POD, their Poincare sections and phase-space reconstructions show evidence of intermittent events somewhat similar to the boundary-layer bursts. Nicolaenko \& She (1990) have made similar observations.

\section{4. 'POD IN DYNAMICAL MODELING}

From our (biased) viewpoint we feel that dynamical modeling is perhaps the most innovative recent use of the POD and of the most interest in the context of the dynamics of coherent structures. We make a distinction between two types of applications: a direct or exact simulation, and a model-based simulation. In the former, one performs a Galerkin projection and the unresolved modes are neglected with the assumption that they are irrelevant to the dynamics; thus one typically must retain many modes [e.g. $O\left(10^{5}\right)$ even for low Reynolds number turbulence]. In the latter, however, one accounts for the unresolved modes by some model and so the dimension can be much reduced. The latter are exactly the type of lowdimensional models we hope will shed light on the dynamics of coherent structures in turbulent flows.

\subsection{Direct Simulations Using the POD}

The motivation behind such studies is the utilization of the fast energy convergence provided by the POD to get well resolved simulations with a lower number of modes than a simulation using Fourier or other types of 
standard decompositions. The questions that are normally studied include: (a) What is the minimal number of modes that reproduces acceptable dynamics? and (b) How do bifurcation sequences differ between a POD simulation and a regular one? Questions relating to dimension are also of interest. Observe that, although the idea of an exact simulation with a smaller (compared to standard) number of modes may seem appealing, the effect of the smaller number may be lost if one is interested in a direct numerical simulation of turbulence since the POD eigenfunctions, in general, are not suited for FFT-type algorithms which offer tremendous savings in computation time.

As we noted in Section 3.4, Sirovich and coworkers (Sirovich 1987, Rodriguez \& Sirovich 1990) have used the Ginzburg-Landau equation as a mathematical model. They study the eigenfunctions, Lyapunov exponents, and bifurcations for the simulated systems and show that, for this model at least, the eigenfunctions change rather slowly with external parameters. This implies that an empirical basis computed for a particular Reynolds number will continue to be advantageous in simulations over a range of Reynolds numbers (see also Chamber et al 1988).

Kirby \& Armbruster (1991) performed simulations of the KuramotoSivashinsky equation. They commented on the need for increased dissipativity in the system to maintain its stability. This fact is well known in various computational disciplines (Anderson et al 1984) and has recently been studied from a mathematical point of view by Foias et al (1991).

\subsection{Models Based on the POD}

The best studied low-dimensional model is that developed by Aubry et al (1988) for the wall region of a turbulent boundary layer. In this model the neglected modes are modeled through a Smagorinsky-type sub-grid-scale model. In addition there is a model that accounts for the evolution of the local mean velocity profile. An introductory review, with some background material on dynamical-systems theory and coherent structures is given in Holmes (1990). Following the original study, which included five (complex) spanwise Fourier modes, a single $\left(k_{1}=0\right)$ streamwise mode, and a single family of eigenfunctions in the wall normal direction, several developments have taken place. Stone (1989) showed that smaller truncations, with 3 and 4 spanwise modes - and certain larger ones, with additional nontrivial streamwise modes - continued to exhibit the heteroclinic cycles and bursting behavior characteristic of the unilateral model, which Aubry et al had shown was remarkably similar to the burst-sweep cycle familiar from experimental studies. Recently the more detailed studies of Aubry \& Sanghi (1989, 1990) (cf Sanghi \& Aubry 1991), which include nontrivial streamwise modes, have added weight to these findings. It is worth noting that 
Armbruster et al's (1988) proof of structural stability of such heteroclinic cycles in $\mathrm{O}(2)$-symmetric systems provides a mathematical foundation and does much to explain their robustness and persistence over a range of parameters and with different truncations (cf Armbruster et al 1989, Holmes 1991).

Aubry et al (1990) scaled Herzog's (1986) eigenfunctions in an attempt to study the effect of boundary-layer modification (by polymer addition or riblets, for example) and found a general shift of parameter values but no new dynamical mechanism or structures, in general agreement with the experimental evidence. Berkooz et al (1991b) in a study prompted by comments of Moffatt (1990), showed that if the cross stream and streamwise velocity components are uncoupled and a dynamical model formulated in which they are allowed to evolve independently, then turbulence having no streamwise variation will decay, as expected. The important point is that the decay is not featureless but ghosts of heteroclinic cycles and the resulting bursting behavior are present. In this study, and in Holmes et al (1991), it is pointed out that the coupled vector-valued empirical eigenfunctions that result from direct application of the POD effectively contain streamwise averages of nontrivial structures in the $k_{1}=0$ streamwise components, and so permit sustained extraction of energy from the mean flow, and hence sustained turbulence. From this viewpoint, use of the POD is similar to a closure assumption.

In another development, Stone \& Holmes $(1990,1991)$ showed that small random and (in some cases) deterministic perturbations, characteristic of coupling to the outer region of the flow, produce distributions of bursting events having exponential tails similar in nature to those observed experimentally (cf Bogard \& Tiederman 1986, Sreenivasan et al 1983, Holmes \& Stone 1991). The thesis of Berkooz (1991b) contained a number of other results related to the original study of Aubry et al (1988). The most relevant in this context is that the low-dimensional models reproduce the energy budget of the full system within acceptable bounds. See also Berkooz et al (1992).

A dynamical model for the jet-annular mixing layer was constructed along the lines of the model for the wall layer by Glauser, George, and coworkers (Glauser, et al 1989, 1990; Zheng \& Glauser 1990). In Glauser et al (1989) and Zheng \& Glauser (1990) dynamical systems describing the interaction of the POD eigenfunctions are constructed and simulated. The result is a nonrecurring system (unlike the wall-layer system) which corresponds to the physical structures in the jet. Glauser and coworkers see it as a cascade mechanism.

Sirovich et al (1990c) proposed an ad-hoc approximate inertial manifold type of approach (Foias et al 1989, Titi 1990, Constantin et al 1989). The 
idea is as follows: suppose that $\left\{\phi_{i}\right\}_{i=1}^{\infty}$ are the POD eigenfunctions and we want to resolve only the subset $\left\{\phi_{i}\right\}_{i=1}^{m}$. Denote by $P$ the projection on span $\left\{\phi_{i}\right\}_{i=1}^{m}$ and let $Q=I-P$. Suppose the original equation is $\dot{u}=F(u)$ with $u=p+q$. We can write

$$
\begin{aligned}
& \dot{p}=P F(p+q), \\
& \dot{q}=Q F(p+q) .
\end{aligned}
$$

The approximate inertial manifold type model is

$$
\dot{p}=P F[p+\Phi(p)],
$$

where the function $q=\Phi(p)$ is obtained by solution of

$$
0=Q F(p+q) \text {. }
$$

The rationale is that unresolved, lower energy modes have a faster time scale (van Kampen 1985) and their variations are not felt by the $p$ 's. Sirovich et al (1990c) apply this to a weakly chaotic system with a truncation which is not as severe as the boundary layer or jet models. In general the algebraic Equation (4.3) is not solved exactly since it may have multiple solutions and an approximation, or truncation, is used. The results of application of this approach to the Ginzburg-Landau equation are given in Sirovich et al (1990c); also see Sirovich (1990).

Finally, we mention the application of the POD to 2-D flows in complex geometries by Deane et al (1991). They considered flows behind a circular cylinder and in a channel with spanwise, rectangular grooves and used a numerically simulated database to produce empirical eigenfunctions which were in turn used to construct low-dimensional ODE models by Galerkin projection. They found that 4-8 mode models could capture the initial (Hopf) bifurcation and loss of stability of the laminar flow rather well, and that, in the case of the grooved channel, the model performed satisfactorily over a range of Reynolds numbers. The cylinder-wake model was more limited in this respect, and studies of fully developed turbulent flows were not carried out for either problem.

\section{RELATION TO OTHER TECHNIQUES}

In this section we comment on the connection between the POD and certain other analysis techniques. We start by describing the connection between the POD and linear stochastic estimation, as applied by Adrian and coworkers in Adrian (1979), Adrian \& Moin (1988), Adrian et al (1987), and Moin et al (1987). This will lead to relations between the POD and conditional sampling techniques such as described in Blackwelder \& 
Kaplan (1976), Bogard \& Tiederman (1986), Johansson \& Alfredsson (1982), Antonia (1981), and references therein. A connection to patternanalysis techniques such as Stretch et al (1990), Townsend (1979), and Ferre \& Girlat (1989) will also be discussed. Recently Aubry et al (1991a) introduced a "new" tool called the bi-orthogonal decomposition. However, a careful examination of their work reveals that their suggestion is in fact a specific case of the rather general POD formulated in Section 2.1. We remark that the formulation of the POD and the results above apply to space, time, or space-time analysis, all depending on the choice of the averaging operator (or equivalently, the measure) as long as the assumptions of Section 2.1 are satisfied. A specific choice of averaging (i.e. a measure concentrated on a finite number of points, as would be encountered in a computer simulation) will produce the decomposition of Aubry et al (199la).

\subsection{Linear Stochastic Estimation}

Suppose one wanted to find the conditional probability density function (CPDF) of $\left[u(x) \mid u\left(x^{\prime}\right)\right]$, where $u\left(x^{\prime}\right)$ may be a multipoint event. For the sake of simplicity of the exposition, we limit ourselves to single-point scalar events. There are many good reasons to seek this CPDF, either for closure models (Pope 1985) or for the sake of producing coherent structures. Suppose, moreover, that one seeks an estimate linear in $u\left(x^{\prime}\right)$, i.e. instead of the full CPDF we want some representative value which would be our best estimate in a sense which we will define below. This is called a linear stochastic estimate. We outline the method of linear stochastic estimation since it is simple and enlightening. We are seeking $A\left(x, x^{\prime}\right)$ such that $A\left(x, x^{\prime}\right) u\left(x^{\prime}\right)$ will be the estimate for $u(x)$, and we want to find $A\left(x, x^{\prime}\right)$ such that

$$
\min _{V\left(x, x^{\prime}\right)}\left\langle\left|u(x)-V\left(x, x^{\prime}\right) u\left(x^{\prime}\right)\right|^{2}\right\rangle
$$

is achieved by $A\left(x, x^{\prime}\right)$. We use the calculus of variations [as in the derivation of (2.2) from (2.1), cf Berkooz 1991b]. A necessary condition will be that, for any $V\left(x, x^{\prime}\right)$, we have

$$
\left.\frac{d}{d \delta}\left\langle\left|u(x)-\left[A\left(x, x^{\prime}\right)+\delta V\left(x, x^{\prime}\right)\right] \cdot u\left(x^{\prime}\right)\right|^{2}\right\rangle\right|_{\delta=0}=0 .
$$

The expression inside the averaging brackets is equal to

$$
\begin{aligned}
\left\{u(x)-\left[A\left(x, x^{\prime}\right)+\delta V\left(x, x^{\prime}\right)\right] \cdot u(x)\right\} & \\
& \left\{u^{*}(x)-\left[A^{*}\left(x, x^{\prime}\right)+\delta V^{*}\left(x, x^{\prime}\right)\right] \cdot u^{*}\left(x^{\prime}\right)\right\} .
\end{aligned}
$$


After taking the average of (5.2), differentiating w.r.t. $\delta$ and evaluating at $\delta=0$, and equating to zcro we get

$$
\begin{aligned}
& 2 \operatorname{Real}\left[V^{*}\left(x, x^{\prime}\right):\left\langle u(x) u^{*}\left(x^{\prime}\right)\right\rangle\right]= \\
& \qquad \operatorname{Real}\left\{V^{*}\left(x, x^{\prime}\right):\left\langle\left[A\left(x, x^{\prime}\right) u\left(x^{\prime}\right)\right] u^{*}\left(x^{\prime}\right)\right\rangle\right\},
\end{aligned}
$$

where : denotes the usual tensor contraction. Therefore we require

$$
\left\langle u(x) u^{*}\left(x^{\prime}\right)\right\rangle=A\left(x, x^{\prime}\right) \cdot\left\langle u\left(x^{\prime}\right) u^{*}\left(x^{\prime}\right)\right\rangle,
$$

and this implies

$$
A\left(x, x^{\prime}\right)=\left\langle u(x) u^{*}\left(x^{\prime}\right)\right\rangle \cdot\left\langle u\left(x^{\prime}\right) u^{*}\left(x^{\prime}\right)\right\rangle^{-1} .
$$

It is natural to ask whether $\left\langle u\left(x^{\prime}\right) u^{*}\left(x^{\prime}\right)\right\rangle$ is invertible at every point $x^{\prime}$ (in the vector case). One can convince oneself that this should typically be the case for a turbulent system. Since here we treat $u$ as a scalar, the inverse of $\left\langle u\left(x^{\prime}\right) u^{*}\left(x^{\prime}\right)\right\rangle$ is just a division.

In (5.6) the average two-point correlation tensor $R\left(x, x^{\prime}\right)$ of Section 2.1 appears with some normalization. Results of Adrian (1979) show that the corrections to the CPDF due to higher order nonlinear terms in $u\left(x^{\prime}\right)$ are small (recall this is the best linear estimate), at least for homogeneous turbulence. Using our previous results (2.9-2.11) we can write

$$
A\left(x, x^{\prime}\right)=\frac{\sum_{i=1}^{\infty} \lambda_{i} \phi_{i}(x) \phi_{i}^{*}\left(x^{\prime}\right)}{\sum_{i=1}^{\infty} \lambda_{i}\left|\phi_{i}\left(x^{\prime}\right)\right|^{2}}=\sum_{i=1}^{\infty} \phi_{i}(x) f_{i}\left(x^{\prime}\right),
$$

where $f_{i}\left(x^{\prime}\right)=\lambda_{i} \phi_{i}^{*}\left(x^{\prime}\right) / \sum_{j=1}^{\infty} \lambda_{j}\left|\phi_{j}\left(x^{\prime}\right)\right|^{2}$. We may interpret $f_{i}\left(x^{\prime}\right)$ as the relative contribution of $\phi_{i}$ to $u\left(x^{\prime}\right)$ on the average. We conclude that linear stochastic estimation is equivalent to assuming that the estimated value of the POD coefficient of the $i$-th mode, given the velocity at $x^{\prime}$, is the average contribution of the $i$-th mode to the velocity of $x^{\prime}$ times the given velocity.

The amazing point is that we get exactly the same result from the simplified PDF model based on the POD introduced at the end of Section 2.6. There we assumed $a_{i} \sim N\left(0, \lambda_{i}\right)$ and that the coefficients $a_{j}$ were independent. Let us compute the estimator $\left\langle u(x) \mid u\left(x^{\prime}\right)\right\rangle$. Since we have an expression for the PDF we can compute this explicitly. Recall from probability theory that if $x_{i} \sim N\left(0, \sigma_{i}^{2}\right)$ for $i=1, \ldots, m$ then

$$
\left\langle x_{i} \mid \sum_{j=1}^{m} x_{j}=C\right\rangle=\frac{\sigma_{i}^{2} C}{\sum_{j=1}^{m} \sigma_{j}^{2}} .
$$

[See the formula for the conditional expectation of joint normal variables in Feller (1957).] Using (5.8), we have 


$$
\left\{a_{i} \phi_{i}\left(x^{\prime}\right) \mid \sum_{j=1}^{\infty} a_{j} \phi_{j}\left(x^{\prime}\right)=u\left(x^{\prime}\right)\right\}=\frac{\lambda_{i}\left|\phi_{i}\left(x^{\prime}\right)\right|^{2} u\left(x^{\prime}\right)}{\sum_{j=1}^{\infty} \lambda_{j}\left|\phi_{j}\left(x^{\prime}\right)\right|^{2}}
$$

which gives

$$
\begin{aligned}
\left\{u(x) \mid u\left(x^{\prime}\right)\right\} & =\frac{\sum_{i=1}^{\infty} \lambda_{i}\left|\phi_{i}\left(x^{\prime}\right)\right|^{2} u\left(x^{\prime}\right) \phi_{i}(x) / \phi_{i}\left(x^{\prime}\right)}{\sum_{j=1}^{\infty} \lambda_{j}\left|\phi_{j}\left(x^{\prime}\right)\right|^{2}} \\
& =\frac{\sum_{i=1}^{\infty} \lambda_{i} \phi_{i}^{*}\left(x^{\prime}\right) \phi_{i}(x) u\left(x^{\prime}\right)}{\sum_{j=1}^{\infty} \lambda_{j}\left|\phi_{j}\left(x^{\prime}\right)\right|^{2}} .
\end{aligned}
$$

This is exactly the same result obtained from linear stochastic estimation (Equation 5.7).

We conclude that the simple PDF model suggested at the end of Section 2.6 results in the best linear estimator of the conditional PDF of velocity, and that linear stochastic estimation may be viewed as a result of the simple PDF model. This reveals the fundamental connection between the POD and linear stochastic estimation. Aside from this we can make the following technical observations based on our previous results:

1. All fields generated by linear stochastic estimation (LSE) possess any closed linear property that all ensemble members share.

2. Suitable averages of LSE events will produce the POD eigenfunctions.

3. All LSE events are linear combinations of POD eigenfunctions.

Finally we remark that one can apply the geometric result from the POD of Section 2.5 to obtain bounds on the probability of rare LSE events.

\subsection{Conditional Sampling}

In this section we indicate a possible connection between the POD and conditional sampling. This exposition is of a speculative nature which we hope might encourage further work in the area. The general conditionalsampling scheme adopted from Antonia (1981) is given in Figure 4. This scheme may be formulated as

$$
R\left(x, \Delta x, \tau_{j}\right)=\frac{1}{N} \sum_{i=1}^{N} c\left(x, t_{i}\right) f\left(x, \Delta x, t_{i}+\tau_{j}\right),
$$

where $c\left(x, t_{i}\right)$ is the conditioning function at a point $x$ in space at time $t_{i}$ which is 1 if a condition is met and 0 otherwise. Once a condition is met, a measurement at a possibly different location (given by $x+\Delta x$ ) and possibly later in time $\left(t_{i}+\tau_{j}\right)$ is added to the averaged ensemble. Conditional averaging has been used in the study of turbulent-nonturbulent interfaces, shear layers perturbed by interaction with another turbulent field, and quasi-periodic or periodic flows (such as those behind a turbine), 


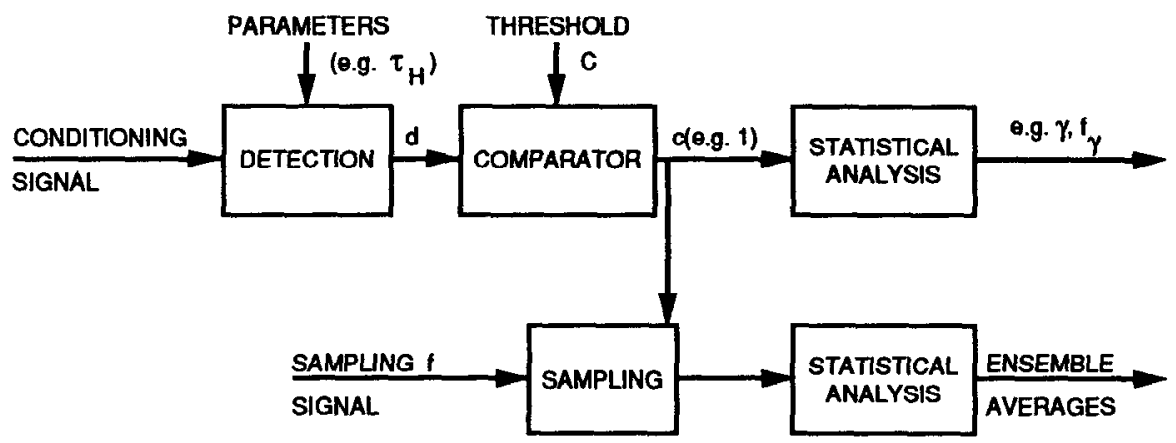

Figure 4 The general scheme of conditional sampling (from Antonia 1981).

and for the study of coherent structures. Our primary interest is in the study of coherent structures. Conditional sampling applied to coherent structures is largely an art: The experimentalist has freedom in defining the threshold and detection criteria and the quantity to be measured. The subjectivity of this procedure may lead to detection of fictitious structures in featureless random fields (Blackwelder \& Kaplan 1976) or may yield the wrong structures (Lumley 1981). This subjectivity precludes the possibility of a rigorous analytic connection, since the POD does not offer this kind of freedom. However, one can study the compatibility between the simple PDF model based on the POD and the results of a conditionalsampling study. This is done through the results on linear stochastic estimation presented in Section 5.1 above. Two comments are in order. First, in order to treat conditional sampling in its full generality, one would have to perform a space-time POD-otherwise the comparison will be limited to conditional sampling with no time delays. Second, the two very strong assumptions made in the process of establishing the correspondence through LSE, namely the independent normal distributions for POD random coefficients and the adequacy of stochastic linear estimations, will prevent us from obtaining decisive conclusions in case of a mismatch. On the other hand, showing such a compatibility would be a welcome contribution to the relation between average quantities such as the POD eigenfunctions and average dynamics of coherent structures. References on conditional sampling are Blackwelder \& Kaplan (1976), Bogard \& Tiederman (1986), Johnansson \& Alfredsson (1982), in addition to the survey paper by Antonia (1981) already referred to, and references therein.

Note that the natural combination of the POD and conditional sampling into a conditional POD based on the condition functions currently used 
might be very illuminating. These conditions also have an interpretation as conditions in phase space and thus are related to the conditional POD mentioned in Section 2.6. Such a combination would give a detailed kinematical description of important processes. If a time-delayed conditional POD were applied, the results might be compared to results of lowdimensional models such as those reviewed in Section 4, thus yielding insight on the dynamics and a comparison for low-dimensional experimental data.

\subsection{Pattern-Recognition Techniques}

In this section we indicate a second possible connection between the POD and pattern-recognition techniques. As above, this section is of a speculative nature. With the advent of digital image processing, pattern recognition has become a vast field (Rosenfeld \& Kak 1982). We limit ourselves to the relatively basic procedures used in fluid problems by Stretch et al (1990), Townsend (1979), and Ferre \& Girlat (1989). Coherent structures were originally identified in flow visualization. The quest for a quantitative procedure for extracting coherent structures and their dynamics is still a subject of research. Pattern-recognition techniques are designed to mimic the human capability of detecting patterns in a noisy medium and thus the hope for their successful application to the task of identifying structures in a flow. We remark that Sirovich \& Kirby (1987) (cf Kirby \& Sirovich 1990) have applied the POD procedure directly to reconstruction of images (of human faces); but the feature-extraction method to be discussed below is somewhat different in spirit.

The basic procedure is as follows: One wants to identify a recurrent pattern in a noisy medium. First one picks a template size and fills it with what is conjectured to look like the coherent structures. The template is then moved around in the data set and after each movement a correlation is computed. Every time the correlation attains a local maximum the corresponding pattern is added to the ensemble, which is averaged to produce a modified reference template. This process is repeated until the template undergoes insignificant further change. The final template is the coherent structure. Once the coherent structure is deduced, one attempts to find regions in space well correlated with this structure and to study their contribution to various statistics.

This again, is a subjective procedure, although Stretch et al (1990) suggest it is a robust one, with the final template being practically independent of the initial condition. Our mathematical understanding of the POD may contribute to a better understanding of the results of patternrecognition applications. Observe the similarity in mission between the pattern-recognition technique and the shot-noise expansion. Both attempt 
to decompose the flow into building blocks (although in pattern recognition we concentrate on regions of the flow with higher correlation with the template). This suggests caution in the interpretation of the resultant template, since, as we saw, any template with a suitable power spectrum might decompose the flow, with an appropriate sprinkling function. This fear is accentuated by the fact that Stretch et al (1990) show a median correlation of only about 0.3 . Based on the shot-noise decomposition one can propose a test for the objectivity of this method to see how well the basic building block is reproduced. Lumley's example (Lumley 1981) is a good starting point for such a quest.

\section{DISCUSSION}

In this paper we have described the proper orthogonal decomposition technique and illustrated its use in the analysis and modeling of turbulent flows. The POD is already a well-established tool for (statistical) data analysis and in data compression. We have argued that it can also be used to address two further types of questions, typified by the following: 1 . Given a complex spatio-temporal signal, what can one determine, via the POD, regarding the system from which it originates? 2. Given a PODGalerkin projection of a PDE such as the Navier-Stokes equation and the resulting finite-dimensional dynamical system, what can one learn about the behavior of the original PDE? We hope that this paper has convinced the reader that recent progress in these areas promises as much or more than the POD has already delivered in its more conventional roles in signal analysis and data compression.

Given a POD analysis of a black box we can say several things. First of all we can determine whether the system exhibits equipartition of energy between different modes. This is immediately apparent from looking at the POD spectrum. If it is a decaying spectrum the system is not equipartitioned and the notion of "more energetic" and "less energetic" modes is meaningful. We can then use finer results (Proposition 2.7 and 2.8) to examine whether the dynamics could have been generated by a compact attractor in phase space. Looking for symmetries (or invariance properties) and using Proposition 2.6 one can, potentially, detect symmetries in the system which might not be apparent otherwise. If the POD eigenfunctions of a given system all share some linear property, using Corollary 2.2 one can deduce that the system as a whole has that property. For example, if measuring in a turbulent flow, say in air, where it is not obvious whether incompressibility is satisfied, a POD analysis will tell us whether the turbulence is divergence free and, if not, to what extent it is not so.

Given a POD analysis which suggests more energetic and less energetic 
modes and the equations of motion one can study the question of the interaction of energetic modes or coherent structures. This is done along the lines of the dynamical studies of the models described in Section 4.2. Note that a successful study is liable to require some physical understanding of the system, although there are some promising mathematical approaches too.

\section{ACKNOWLEDGMENTS}

Support for this work was provided by the Air Force Office of Scientific Research under grant AFOSR 89-0226.

\section{Literature Cited}

Adrian, R. J. 1979. Conditional eddies in isotropic turbulence. Phys. Fluids 22(11): 2065-70

Adrian, R. J., Moin P. 1988. Stochastic estimation of organized turbulent structure: homogeneous shear flow. J. Fluid Mech. 190: 531-59

Adrian, R. J., Moin, P., Moser, R. D. 1987. Stochastic estimation of conditional eddies in turbulent channel flow. In $C T R$, Proc. Summer Prog. 1987, pp. 7-20

Algazi, V. R., Sakrison, D. J. 1969. On the optimality of the Karhunen-Loève expansion. IEEE Trans. Inform. Theory 15: 31921

Anderson, D. A., Tannehill, J. C., Pletcher, R. H. 1984. Computational Fhid Mechanics and Heat Transfer. Washington, DC: Hemisphere

Andrews, C. A., Davies, J. M., Schwartz, G. R. 1967. Adaptive data compression. Proc. IEEE 55: 267-77

Antonia, R. A. 1981. Conditional sampling in turbulence measurement. Annu. Rev. Fluid. Mech. 13: 131-56

Armbruster, D., Guckenheimer, J., Holmes, P. 1988. Heteroclinic cycles and modulated travelling waves in systems with $O(2)$ symmetry. Physica D 29: 257-82

Armbruster, D., Guckenheimer, J., Holmes, P. 1989. Kuramoto-Sivashinksy dynamics on the center unstable manifold. SIAM J. Appl. Math. 49: 676-91

Aubry, N., Sanghi, S. 1989. Streamwise and cross-stream dynamics of the turbulent wall layer. In Chaotic Dynamics in Fluid Mechanics, Proc. 3rd Joint ASCE-ASME Mech. Conf., UCSD, La Jolla, CA, July 9-12, ed. K. N. Ghia, U. Ghia. New York: ASME

Aubry, N., Sanghi, S. 1990. Bifurcations and bursting of streaks in the turbulent wall layer. In Turbulence and Coherent Structures, Proc. Grenoble (France) Conf. on Organized Structures and Turbulence in Fluid Mech., 18-21 Sept., ed. M. Lesieur , O. Métais, pp. 227-51. Dordrecht: Kluwer Aubry, N., Holmes, P., Lumley, J. L., Stone, E. 1988. The dynamics of coherent structures in the wall region of a turbulent boundary layer. J. Fluid Mech. 192: 11573

Aubry, N., Lumley, J. L., Holmes, P. 1990. The effect of modeled drag reduction on the wall region. Theoret. Comput. Fluid Dynam. 1: 229.48

Aubry, N., Guyonnet, R., Lima, R. 1991a. Spatio-temporal analysis of complex signals: theory and applications. $J$. Stat. Phys. 64(3/4): 683-793

Aubry, N., Lian, W.-L., Titi, E. S. 1991b. Preserving symmetries in the proper orthogonal decomposition. Tech. Rep.9135, MSI

Bakewell, P., Lumley, J. L. 1967. Viscous sublayer and adjacent wall region in turbulent pipe flows. Phys. Fluids 10: 188089

Ball, K. S., Sirovich, L., Keefe, L. R. 1991. Dynamical eigenfunction decomposition of turbulent channel flow. Int. J. Numerical Methods Fluids 12: 585-604

Bartlet, H., Lohmann, A. W., Wirnitzer, B. 1984. Phase and amplitude recovery from bispectra. Appl. Opt. 23: 3121-29

Berkooz, G. 1990a. Observations on the proper orthogonal decomposition. In Studies in Turbulence, ed. T. B. Gatski, S. Sarkar, C. G. Speziale, pp. 229-47. New York: Springer-Verlag

Berkooz, G. 1990b. Some examples concerning the POD. Preprint 
Berkooz, G. 1991a. Statistical analysis of phase portraits: bifurcations and perturbations of limit cycles. Preprint

Berkooz, G. 1991b. Turbulence, coherent structures and low dimensional models. PhD thesis. Cornell Univ.

Berkooz, G., Titi, E. 1992. The POD systems with symmetry and harmonic analysis. In preparation

Berkooz, G., Elezgaray, J., Holmes, P. 1991a. Coherent structures in random media and wavelets. Proc. DARPA/ ONERA Workshop on Wavelets and Applications, Princeton, June 1991, Physica $D$ (to appear)

Berkooz, G., Holmes, P., Lumley, J. L. $1991 \mathrm{~b}$. Intermittent dynamics in simple models of the wall layer. J. Fluid Mech. 230: 75-95

Berkooz, G., Holmes, P., Lumley, J. L. 1992. On the relations between low dimensional models and the dynamics of coherent structures in the wall layer. Preprint

Blackwelder, R. F., Kaplan, R. E. 1976. On the wall structure of the turbulent boundary layer. J. Fluid Mech. 76: 89-112

Bogard, D. G., Tiederman, W. G. 1986. Burst detection with single single-point velocity measurements. J. Fluid Mech. 162: 389-413

Brillinger, D. R., Rosenblatt, M. 1967. Asymptotic theory of estimates of $\mathrm{kth}$ order spectra. In Spectral Analysis of Time Series, ed. B. Harris. New York: Wiley

Chambers, D. H., Adrian, R. J., Moin, P. Stewart, D. S., Sung, H. J. 1988. Karhunen-Loève expansion of Burgers' model of turbulence. Phys. Fluids 31(9): 2573-82

Constantin, P., Foias, C. 1989. NavierStokes Equations. Chicago: Chicago Univ. Press

Constantin, P., Foias, C. Temam, R., Nicolaenko, B. 1989. Integral Manifolds and Inertial Manifolds for Dissipative Partial Differential Equations. New York: Springer-Verlag

Deane, A. E., Sirovich, L. 1991. A computational study of Rayleigh-Bénard convection. Part I: Rayleigh number scaling. J. Fluid Mech. 222: 231-50

Deane, A. E., Kevrekidis, I. G., Karniadakis, G. E., Orszag, S. A. 1991. Low dimensional models for complex geometry flows: application to grooved channels and circular cylinder. Phys. Fluids A 3: 2337-54

Farge, M. 1992. The wavelet transform and its applications to fluid mechanics. Annu. Rev. Fluid Mech. 24: 395-457

Feller, W. 1957. An Introduction to Probability Theory and Its Applications. New York: Wiley

Ferre, J. A., Girlat, F. 1989. Pattern-recognition analysis of the velocity field in plane turbulent wakes. J. Fluid Mech. 198: $27-64$

Foias, C., Témam, R. 1989. Gevrey class regularity for the solutions of the NavierStokes equations. J. Func. Anal. 87: 359 69

Foias, C., Sell, G. R., Titi, E. S. 1989. Exponential tracking and approximation of inertial manifolds for dissipative equations. $J$. Dyn. Differ. Equ. 1: 199-224

Foias, C., Manley, O., Sirovich, L. 1990. Empirical and Stokes eigenfunctions and the far dissipative turbulent spectrum. Phys. Fluids A 2: 464-67

Foias, C., Jolly, M. S., Kevrekidis, I. G., Titi, E.S. 1991. Dissipativity of numerical schemes. Nonlinearity 4: (to appear)

Gay, D. H., Ray, W. H. 1986. Identification and control of linear distributed parameter systems through the use of experimentally determined singular functions. Proc. IFAC Symp. Control of Distributed Parameter Systems, Los Angeles, 30 June-2 July 1986, ed. H. E. Rauch, pp. 173-79. Oxford/New York: Pergamon

Gay, D. H., Ray, W. H. 1988. Application of singular value methods for identification and model based control of distributed parameter systems. Proc. IFAC Workshop on Model-Based Process Control, Atlanta, GA, June 1988, ed. J. T. McAvoy, Y. Arkun, E. Zafiriou, pp. 95-102. Oxford/ New York: Pergamon

Glauser, M. N., George, W. K. 1987a. Orthogonal decomposition of the axisymmetric jet mixing layer including azimuthal dependence. In Advances in Turbulence, ed. G. Comte-Bellot, J. Mathicu. New York: Springer-Verlag

Glauser, M. N., George, W. K. $1987 \mathrm{~b}$. Orthogonal decomposition of the axisymmetric jet mixing layer utilizing cross-wire vclocity measurements. In 6 th Symp. Turbulent Shear Flows, 1987

Glauser, M. N., Leib, S. J., George, W. K. 1987. Coherent structures in the axisymmetric turbulent jet mixing layer. In Turbulent Shear Flows 5, ed. F. Durst, B. E. Launder, J. L. Lumley, F. W. Schmidt, J. H. Whitelaw. New York: SpringerVerlag

Glauser, M. N., Zheng, X., Doering, C. R. 1989. The dynamics of organized structures in the axisymmetric jet mixing layer. In Studies in Turbulence, ed. T. Gatski, S. Sarkar, C. Speziale, pp. 207-22. New York: Springer-Verlag

Glauser, M. N., Zheng, X., George W. K. 1990. The streamwise evolution of coherent structures in the axisymmetric jet mixing layer. In The Lumley Symposium: Recent Developments in Turbulence, Newport News, VA, Nov. 1990, ed. T. Gatski 
et a1., pp. 207-22. New York: SpringerVerlag

Glezer, A., Kadioglu, A. J., Pearlstein, A. J. 1989. Development of an extended proper orthogonal decomposition and its application to a time periodically forced plane mixing layer. Phys. Fluids A 1: 1363-73

Herzog, S. 1986. The large scale structure in the near wall region of a turbulent pipe flow. PhD thesis. Cornell Univ.

Holmes, P. J. 1990. Can dynamical systems approach turbulence? In Whither Turbulence? Turbulence at the Crossroads, ed. J. L. Lumley, Lect. Notes Phys. 357: 195249

Holmes, P. J. 1991. Symmetries, heteroclinic cycles and intermittency in fluid flow. Proc. I. M. A. Workshop on Dynamical Theories of Turbulence (Minnesota, May 29-June 2, 1990) New York: SpringerVerlag. In press

Holmes, P. J., Stone, E. 1991. Heteroclinic cycles, exponential tails and intermittency in turbulence production. In Studies in Turbulence, ed. T. Gatski, S. Sarkar, C. Speziale, pp. 179-89. New York: SpringerVerlag

Holmes, P. J., Berkooz, G., Lumley, J. L. 1991. Turbulence, dynamical systems and the unreasonable effectiveness of empirical cigenfunctions. In Proc. ICM-90, K yoto, Japan. New York: Springer-Verlag

Hopf, E. 1957. On the application of functional calculus to the statistical theory of turbulence. In Proc. Symp. Appl. Math. Am. Math. Soc.

Jimenez, J., Moin, P. 1991. The minimal flow unit in near-wall turbulence. $J$. Fluid Mech. 225: 213-20

Johansson, A. V., Alfredsson, P. H. 1982. On the structure of turbulent channel flow. J. Fhid Mech. 122: 295-314

Karhunen, K. 1946. Zur spektral theorie stochastischer prozesse. Ann. Acad. Sci. Fennicae Ser. A1: 34

Keefe, L., Moin, P., Kim, J. 1987. The dimension of an attractor in turbulent Poiseuille flow. Bull. Am. Phys. Soc. 32: 2026

Kim, J., Moin, P., Moser, R. J. 1987. Turbulence statistics in a fully developed channel flow at low Reynolds number. $J$. Fluid Mech. 177: 133-66

Kirby, M., Armbruster, D. 1991. Reconstructing phase space for PDE simulations. Preprint

Kirby, M., Sirovich, L. 1990. Application of the Karhunen-Loeve procedure for the characterization of human faces. IEEE Trans. Pattern Anal. and Mach. Intell. 12: 103-8

Kirby, M., Boris, J., Sirovich, L. 1990a. An eigenfunction analysis of axisymmetric jet flow. J. Comput. Phys. 90(1): 98-122
Kirby, M., Boris, J., Sirovich, L. 1990b. A proper orthogonal decomposition of a simulated supersonic shear layer. Int. $J$. Numerical Methods Fluids 10: 411-28

Kline, S. J., Reynolds, W. C., Schraub, F. A., Rundstadler, P. W. 1967. The structure of turbulent boundary layers. J. Fluid Mech. 30: 741-73

Kosambi, D. D. 1943. Statisties in function space. J. Indian Math. Soc. 7: 76-88

Liepmann, H. W. 1952. Aspects of the turbulence problem. Part II Z. Angew. Math. Phys. 3: 407-26

Lii, K. S., Rosenblatt, M., Van Atta, C. 1976. Bispectral measurements in turbulence. J. Fluid Mech. 77: 45-62

Liu, J. T. C. 1988. Contributions to the understanding of large scale coherent structures in developing free turbulent shear flows. Adv. Appl. Mech. 26: 183209

Loève, M. 1945. Functions aleatoire de second ordre. C. R. Acad. Sci. Paris 220

Lumley, J. L. 1967. The structure of inhomogeneous turbulence. In Atmospheric Turbulence and Wave Propagation, ed. A. M. Yaglom, V. I. Tatarski, pp. 166-78. Moscow: Nauka

Lumley, J. L. 1971. Stochastic Tools in Turbulence. New York: Academic

Lumley, J. L. 1981. Coherent structures in turbulence. In Transition and Turbulence, ed. R. E. Meyer, pp. 215-42. New York: Academic

Lumley, J. L. 1989. The state of turbulence research. In Advances in Turbulence, ed. W. K. George, R. Arndt, pp. 1-10. Washington, DC: Hemisphere

Matsuoka, T., Ulrych, T. L. 1984. Phase estimation using the bispectrum. Proc. IEEE 72: 1403-22

McComb, W. D. 1990. The Physics of Turbulence. Oxford: Clarendon

Meyer, Y. 1987. Ondelettes, fonctions splines at analyses graduées. Rapp. CER$E M A D E$ 8703, Univ. Paris-Dauphane

Moffatt, H. K. 1990. Fixed points of turbulent dynamical systems and suppression of nonlinearity. In Whither Turbulence? Turbulence at the Crossroads, ed. J. L. Lumley, Lect. Notes Phys. 357: 250-57

Moin, P., Moser, R. D. 1989. Characteristiceddy decomposition of turbulence in a channel. J. Fluid Mech. 200: 471-509

Moin, P., Adrian, R. J., Kim, J. 1987. Stochastic estimation of organized structures in turbulent channel flow. In 6th Turbulence Shear Flow Symp., Toulouse, France, pap. 16-9

Monin, A. S., Yaglom, A. M. 1987. Statistical Fluid Mechanics, ed. J. L. Lumley. Cambridge, MA: MIT Press

Nicolaenko, B., She, Z.-S. 1990. Temporal 
intermittency and turbulence production in the Kolmogorov flow. In Topological Fluid Mechanics, ed. H. K. Moffatt, p. 256. Cambridge: Cambridge Univ. Press

Novikov, E. A. 1963. Variability of energy dissipation rate in a turbulent flow and the energy distribution over the spectrum. Prikl. Mat. Mekh. 27(5): 944 46

Obukhov, A. M. 1954. Statistical description of continuous fields. Tr. Geophys. Int. Akad. Nauk. SSSR 24: 3-42

Papoulis, A. 1965. Probability, Random Variables, and Stochastic Processes. New York: McGraw-Hill

Park, H., Sirovich, L. 1990. Turbulent thermal convection in a finite domain: Part II numerical results. Phys. Fluids $A$ 2(9): 1659-68

Perrier, V., Basdevant, C. 1989. Periodic wavelet analysis: a tool for inhomogeneous field investigation. Theory and Algorithms. Rech. Aérosp. No. 1989-3, pp. 54-67

Platt, N., Sirovich, L., Fitzmaurice, N. 1991. An investigation of chaotic Kolmogorov flows. Phys. Fluids 3(4): 681-96

Pope, S. B. 1985. Pdf methods for turbulent reactive flows. Prog. Energy Combust. Sci. 11: 119-92

Pougachev, V. S. 1953. General theory of the correlations of random functions. Izv. Akad. Nauk. SSSR, Ser. Mat. 17: 1401-2

Preisendorfer, R. W. 1988. Principal Component Analysis in Meteorology and Oceanography. Amsterdam: Elsevier

Promislow, K. 1991. Time analyticity and Gevrey regularity for solutions of a class of dissipative pde's. Nonlinear. Anal. 16(11): 959-80

Reynolds, O. 1894. On the dynamical theory of incompressible viscous fluids and the determination of the criterion. Phil. Trans. R. Soc. London 186: 123-61

Rice, S. O. 1944. Mathematical analysis of random noise. Bell Sys. Tech. J. 23: 282332

Riesz, F., Nagy, B. 1955. Functional Analysis. New York: Ungar

Robinson, S. K. 1991. Coherent motions in the turbulent boundary layer. Annu. Rev. Fluid Mech. 23: 601-39

Rodriguez, J. D., Sirovich, L. 1990. Lowdimensional dynamics for the complex Ginsburg-Landau equation. Physica $D 43$ : 77-86

Rosenfeld, A., Kak, A. C. 1982. Digital Picture Processing. New York: Academic

Ruelle, D., Takens, F. 1971. On the nature of turbulence. Comm. Math. Phys. 20: $167-92 ; 23: 343-44$

Sanghi, S., Aubry, N. 1991. Mode interaction models for near wall turbulence. Levich Inst. Preprint
Sirovich, L. 1987. Turbulence and the dynamics of coherent structures, parts iiii. Quart. J. Appl. Math. 45(3): 561-90

Sirovich, L. 1989. Chaotic dynamics of coherent structures. Physica $D$ 37: 12645

Sirovich, L. 1990. Empirical eigenfunctions and low dimensional systems. Cent. Fluid Mech. Rep. 90-202, Brown Univ.

Sirovich, L., Deane, A. E. 1991. A computational study of Rayleigh Bénard convection. Part II. Dimension considerations. J. Fluid Mech. 222: 251-65

Sirovich, L., Kirby, M. 1987. Low-dimensional procedure for the characterization of human faces. J. Opt. Soc. Am. A 4: 519-24

Sirovich, L., Knight, B. W. 1985. The eigenfunctions problem in higher dimensions: asymptotic theory. Proc. Natl. Acad. Sci. 82: 8275-78

Sirovich, L., Park, H. 1990. Turbulent thermal convection in a finite domain: Part I. Theory. Phys. Fluids A 2(9): 164958

Sirovich, L., Ball, K. S., Keefe, L. R. 1990a. Plane waves and structures in turbulent channel flow. Phys. Fluids $A$ 2(12): 2217 26

Sirovich, L., Kirby, M., Winter, M. 1990 b. An eigenfunction approach to large scale transitional structures in jet flow. Phys. Fluids $A$ 2 (2): 127-136

Sirovich, L., Knight, B. W., Rodriguez, J. D. 1990c. Optimal low-dimensional dynamical approximations. Quart. J. Appl. Math. 48(3) : $535-48$

Sreenivasan, K. R., Narashima, R., Prabhu, A. 1983. Zero-crossings in turbulent signals. J. Fluid Mech. 137: 251-72

Stonc, E. 1989. A study of low dimensional models for the wall region of a turbulent boundary layer, $\mathrm{PhD}$ thesis. Cornell Univ.

Stone, E., Holmes, P. J. 1990. Random perturbations of heteroclinic cycles. SIAM J. Appl. Math. 50(3) : 726-43

Stone, E., Holmes, P. J. 1991. Unstable fixed points, heteroclinc cycles and exponential tails in turbulence production. Phys. Lett. A 155: 29-42

Stretch, D., Kim, J., Britter, R. 1990. A conceptual model for the structure of turbulent channel now. In Notes for Boundary Layer Structure Workshop, ed. S. Robinson, NASA Langley, Aug 28-30. See also Stretch, D. 1991. Automated pattern eduction from turbulent flow diagnostics. 1991 Annu. Res. Briefs, Cent. for Turb. Res., Stanford Univ., pp. 145-57

Témam, R. 1988. Infinite-Dimensional Dynamical Systems in Mechanics and Physics. New York: Springer-Verlag

Tennekes, H., Lumley, J. L. 1972. A First 
Course in Turbulence. Cambridge, MA: large eddies in a wake and in a boundary MIT Press

layer. J. Fluid Mech. 95: 551

Titi, E. S. 1990. On approximate inertial manifolds to the Navier-Stokes equations. J. Math. Anal. Appl. 149: 540-57

van Kampen, N. G. 1985. Elimination of fast variables. Phys. Rep. 123: 69-160

Townsend, A. A. 1956. The Structure of Turbulent Shear Flow. Cambridge, UK: Cambridge Univ. Press

Zheng, X., Glauser, M. N. 1990. A low dimensional description of the axisymmetric jet mixing layer. $A S M E$ Comput. Eng. 2: 121-27

Townsend, A. A. 1979. Flow patterns of 